\title{
A Ten year Clinicopathological Study of Female Genital Tuberculosis and Impact on Fertility
}

\author{
Mondal SK, 'Dutta TK' \\ 'Department of Pathology, Medical College, Kolkata-73, INDIA
}

\section{ABSTRACT}

Introduction: To determine the histological pattern of involvement, clinical presentation, impact on fertility in women with genital tuberculosis.

Methods: A total number of 68 cases of gynaecological tuberculosis affecting different parts of female genital tract from 56 patients were selected. The age range of the patients was 17-36 years with mean age of 25.6 years. The diagnostic procedures used included endometrial curettage and biopsy, histopathological examination, culture and Mycobacterium Tuberculosis Polymerase Chain Reaction (MTB PCR), laparoscopy, Hysterosalpingography (HSG) and Ultrasonography (USG). Most of the specimens received were biopsies of endometrial curettage for evaluation of infertility. In 7 cases, specimens of total hysterectomy with bilateral salpingo-oophorectomy were submitted with lesions involving multiple sites.

Results: Patients presented with infertility (65-70\%), pelvic/abdominal pain (50-55\%), and menstrual disturbances (20-25\%). Tuberculosis involved the endometrium in $55.88 \%$, tubes in $23.53 \%$, ovaries in $14.71 \%$ and cervix in $5.88 \%$ of the 68 cases. The endometrium( 38 cases)was in the proliferative phase in 31 cases $(81.57 \%)$, secretory phase in 4 cases $(10.52 \%)$ and it was atrophic in 3 cases $(7.89 \%)$. Caseation was present in 9 out of 68 cases and Ziehl-Neelsen (ZN) stain revealed Acid Fast Bacilli (AFB) in tissue sections in only 4 cases . After therapy, 9 patients conceived of which 8 suffered spontaneous abortions. Only one patient had a successful pregnancy and the baby was born through caesarean section.

Conclusions: Genital tuberculosis is an important cause of female infertility in developing countries like India, Nepal, Bangladesh and Pakistan. Successful uterine pregnancy is rare after treatment and chances of ectopic pregnancy are high.

Key Words: female infertility, genital tuberculosis, histology

\section{INTRODUCTION}

Genital tuberculosis (TB) predominantly affects individuals below 40 years of age and peak age frequency ranges between 21 to 30 year of age. ${ }^{1}$ The disease is responsible for $5 \%$ of all female pelvic infections and occurs in $10 \%$ cases of pulmonary tuberculosis. Gupta et al, studied 40 infertile women with genital tuberculosis and found pulmonary tuberculosis in 9 of them and positive Mantoux test results in two 
Mondal et al. A Ten year Clinicopathological Study of Female Genital Tuberculosis and Impact on Fertility

patients. ${ }^{2}$ Tuberculosis of female genital tract although uncommon in western world, is still prevalent in developing countries like India, Pakistan. ${ }^{3}$ Most cases were detected in infertile women in the reproductive age group and sterility was the predominant motive for consultation. ${ }^{4,5}$

Genital tuberculosis of women occurs secondary to primary disease in the lungs, lymph nodes, urinary tract, bones, joints, and bowel and spreads to genital organs. The spread is usually by haematogenous or lymphatic route. Sexual transmission of the disease is also documented but direct spread from other intraperitoneal foci is very rare.

\section{METHODS}

A prospective study was carried out on female genital tuberculosis in tertiary hospital based study done in Medical College and Hospital, Kolkata, India from July, 1998 to June, 2008. Our institutional approval and patients' consent was taken. Females who were suffering from genital tuberculosis were included in this study. Detailed clinical information, HSG/USG findings and relevant investigations were recorded from the case sheets. These included age of the patients, signs and symptoms, routine haematological check up, chest $x$-ray, Mantoux/Purified Protein Derivative (PPD) skin tests etc. The diagnostic procedures used to detect the lesions included histopathological examination of tissue biopsies, culture and MTB PCR detection of bacteria in tissue and fluid (peritoneal and menstrual). HSG was done in 38 cases and USG in 27 cases. Most of the specimens (43 cases) received were biopsies of endometrial curettage for evaluation of infertility. Patients presented with either tubal involvement (10 cases) or with tubo-ovarian mass (six cases) and subsequently underwent surgical removal. In 7 cases, specimens of total hysterectomy with bilateral salpingoophorectomy were submitted with lesions involving multiple sites. Tissue sections were processed and paraffin blocks were made. Slides were stained by Haematoxylin andEosin (HandE) stain, and Ziehl-Neelsen (ZN) stain routinely. Periodic Acid Schiff (PAS) and other special stains like Gomori's methenamine silver stain were done when necessary to exclude fungal etiology (five cases).

Exclusion criteria were females with proven diagnosis of other causes of infertility. Patients, who had granulomas on biopsy but later on proved to be of nontuberculous origin, were also excluded from our study. Patients who showed negative results for detection of Mycobacterium tuberculosis either in tissue sections (ZN stain) or in culture or in molecular methods (PCR) were excluded from the present study.
For extraction of DNA, decontaminated sediment $(200 \mu \mathrm{l})$ was incubated in a lysis buffer containing 20 $\mathrm{mM}$ Tris $/ \mathrm{HCl}(\mathrm{pH}$ 8.3. DNA was purified in phenol and DNA extract $(10 \mu \mathrm{l})$ was mixed with primers $(0.4 \% \mu \mathrm{M})$ and other reagents. After that amplification was carried out for 30 cycles; each consisting of denaturation at $94^{\circ} \mathrm{C}$ for 2 minutes, annealing at $60{ }^{\circ} \mathrm{C}$ for 2 minutes and extension at $72{ }^{\circ} \mathrm{C}$ for 2 minutes. Two pairs of oliginucleotide primers were used for molecular diagnosis. A $123 \mathrm{bp}$ fragment corresponding to a specific mycobacterium tuberculosis complex sequence which was the insertion sequence 6110(IS 6110) and a 383 bp DNA fragment encoding part of the $65 \mathrm{kD}$ mycobacterial surface antigen were amplified by PCR.

Data was compiled and statistical analysis was done using Microsoft Excel. Mean, Median and other relevant statistical data were obtained.

\section{RESULTS}

Total 68 case of genital tuberculosis were observed in the ten year duration of study. Patients presented with infertility (65-70\%), pelvic/abdominal pain (50-55\%), and menstrual disturbances (20-25\%). Hysterosalpingography (HSG) was performed in 38 cases and revealed abnormality either in fallopian tube (11 cases) or in uterine cavity/endometrium (two cases). The findings in fallopian tube were tubal dilatation (7/11), closed fimbrial ends (6/11), peritubal adhesion (4/11), and irregularity of tubal outline due to mucosal ulceration (2/11). Endometrial synechiae was detected in two cases. Ultrasonographic examination was done in 27 cases and findings were adnexal mass (16/27), dilated/irregular fallopian tube $(11 / 27)$, peritubal adhesions (8/27), ascites (9/27) and thickened peritoneum (3/27).

Tuberculous inflammation was seen in endometrium in 38 cases $(55.88 \%)$. The cervix was the least common site (four cases, $5.88 \%$ ). Caseation was present in nine of 68 cases $(13.23 \%)$ and acid-fast bacilli were found in tissue sections in four cases $(5.88 \%$ ) (Table 1 ). No case of TB vulva and vagina was seen in this study.

The principal histological findings in tuberculous endometritis (38 cases) were presence of the epithelioid cell granulomas. Granulomas were small, isolated lesions scattered throughout the endometrium in most of the cases (31/38). Aggregates of epithelioid histiocytes and with a peripheral collar of lymphocytes were found scattered in the functionalis layer of endometrium (Figure 1). Caseation was found very rarely (one case) in the endometrium and the patient was post-menopausal. Multinucleated giant cells of both Langhans and foreign body type were present in seven cases with disruption of endometrial glands in 
Mondal et al. A Ten year Clinicopathological Study of Female Genital Tuberculosis and Impact on Fertility

two cases. Plasma cells were present in three cases of tuberculous endometritis and indicated secondary infection. Granulomas in different stages were found in eight cases of tuberculous endometritis.

Grossly, fallopian tubes in tuberculous salpingitis (16 cases) appeared enlarged and slightly oedematous with irregular external surfaces due to adhesions. Fallopian tubes were involved bilaterally in 13 cases and fimbrial ends were closed in six cases. In three cases, the fimbria were everted with a patent orifice, imparting the characteristic "tobacco pouch" appearance. Cut sections revealed serosanguineous fluid (five cases) or blood (one case) or caseous material (three cases) with diffuse or focal mucosal ulceration. The lumen contained clear fluid in four cases (hydrosalpinx) and pus (pyosalpinx) in three cases. The histology of tuberculous salpingitis was not always specific. In the early stage (five cases), the features were of chronic salpingitis with occasional non-caseating granulomas. In this stage, plical adhesions gave the appearance of follicular salpingitis.In advanced stages (11 cases), single or multiple confluents epithelioid granulomas were present in the lamina propria (Figure 2). The tubercles and chronic inflammation involved the muscularis (two cases) and serosa (one case) infrequently (Figure 3 ). Caseating granulomas were present in five out of 16 cases of tuberculous salpingitis and two cases showed AFB in tissue sections.

Ovarian tuberculosis (10 cases) was usually a sequel of a tubal lesion and presented as a tubo-ovarian mass (Figure 4). Histological examination at the stage of peri-oophoritis (2/10) showed fibrinous exudates on the surface but with extension to the ovarian parenchyma, a multilocular thick-walled abscess developed.This usually involved both the ovary and tube and microscopy revealed necrosis, granulation tissue formation and neutrophilic infiltration. In the long standing cases (8/10), fibrotic adhesions between ovary and surrounding structures, especially fallopian tube occurred; which might play a role in the sterility in these patients. The granulomas were present in the cortical surface (7/10) though diffuse involvement was also seen (3/10). Microscopically, caseation was present in three out of 10 cases of ovarian tuberculosis and one case revealed AFB on ZN stain (Figure 5).

Cervical tuberculosis (four cases) was almost always secondary to involvement elsewhere in female genital tract. Macroscopically, the cervix was red, enlarged, friable and ulcerated which led to misdiagnoses of cervical cancer clinically in two cases. The mucosa of the endocervical canal was commonest site (three cases) of involvement. The granulomas consisted of epithelioid cells and lymphocytes as in other sites of genital tuberculosis but caseation was absent.
Epithelioid granulomas suggestive of tuberculosis were found microscopically in 73 cases, of which 68 cases were proven for mycobacterium tuberculosis either by detection of AFB in tissue sections, or by mycobacterial culture on Lowenstein Jensen medium/ BACTEC culture or by MTB PCR. In four cases, diagnoses were confirmed by presence of AFB in tissue sections on ZN stain and in 37 cases by mycobacterial culture (BACTEC /LJ) of tissue or menstrual/ peritoneal fluid. In the remaining 27 cases, the bacteria did not grow in culture and was detected by identification of particular DNA sequence by MTB PCR on agarose gel electrophoresis (Figure 6). The other five cases were excluded from this study due to lack of definite diagnostic evidence. Women with genital tuberculosis were treated with long-term combined antituberculous drug therapy. Surgical treatment was employed for macro-lesional forms which were resistant to medical treatment .Conservative surgery was attempted in nine patients and radical surgery in seven patients.Tubal reconstructive surgery was performed in two patients. After therapy; nine women conceived (seven tubal pregnancies and only two uterine pregnancies), some with multiple pregnancies.Out of the nine pregnancies eight suffered spontaneous abortion. Only one patient had a successful pregnancy who delivered a baby through Caesarean section.

\section{DISCUSSION}

Tuberculosis remains a major global health problem and most of these are pulmonary TB.

Actual frequency of female genital tuberculosis is unknown despite different published data from various countries as it is often discovered incidentally or remain 'undetected' in symptomless patients. ${ }^{6}$ More than $90 \%$ of patients of female genital TB were under 40 years of age which indicated the hormone dependent nature of the disease and corroborated other studies. ${ }^{7,8}$ The frequency of genital tuberculosis was $0.002 \%$ of all patients admitted for gynaecologic diseases. ${ }^{9} \quad$ In postmenopausal women genital tuberculosis is rare and seen in $1 \%$ of patients with postmenopausal bleeding. ${ }^{10}$ The exact cause of low incidence of the disease in this age group is not known. Most authors believe that an atrophic endometrium is a poor milieu for the growth of mycobacterium tuberculosis. Since 1950, however, the peak incidence has shifted to the perimenopause as infertility has been superseded by abnormal uterine bleeding and pelvic pain as dominant complaint in the developed countries. ${ }^{9,11}$ Genital tuberculosis is usually an indolent infection and takes years to manifest clinically after initial seeding.

Genital TB may be suspected from the clinical presentation, radiological findings (USG, HSG etc) and 
Mondal et al. A Ten year Clinicopathological Study of Female Genital Tuberculosis and Impact on Fertility

other investigations like positive Mantoux test, high sedimentation rate, tuberculous foci on chest $x$-ray film. On pelvic examination of patients with genital TB, Saracoglu and collegues found that no abnormaliy in $43 \%$ of cases, adnexal mass $(23.6 \%)$, myoma-like lesion $(23.6 \%)$, adnexal tenderness $(4.2 \%)$, irregular uterus $(1.4 \%)$ and cervical polyp $(1.4 \%)$.

In tuberculous endometritis, caseation is rare in women of reproductive age group. ${ }^{12}$ In our study; caseation was found in 1 case only, when the patient had amenorrhoea due to menopause.

In that case the granulomas remained within the endometrial tissue long enough for caseation to develop, as there was no periodical loss of endometrium of menstruation. The disease process has to regenerate after menstrual shedding from the basal layer of endometrium with start of each menstrual cycle and the granulomas become well developed and numerous as the cycle progresses. So, biopsy is recommended immediately before the menstrual phase as the granulomas get longest possible time to develop and greater chance of providing accurate diagnosis. In our study, the endometrial glands were usually unaffected but showed poor response to ovarian hormones which might have contributed to the infertility. Nogales-Ortiz et al found abundant lymphoid follicles without granuloma in the endometrium in patients who have had both fallopian tubes involved. ${ }^{13}$ When TB affects the female genital tract, the fallopian tube is involved in nearly all patients; involvement of the endometrium is usually secondary to tubal disease. But in this study, number of endometrial TB (38 case) is more than fallopian tube TB (16 cases).This might be due to sample biasness, as most of the specimens we received were endometrial biopsies for evaluation of infertility.

The tubal mucosa is most favorable nidus to blood borne tubercle bacilli and endosalpingitis, usually bilateral, is the earliest lesion with transluminal spread to ovary and uterus. The gross pathology of tuberculous endosalpingitis is variable and bilateral involvement is common, owing to typical tendency of the tubercle bacillus to attack each one of bilateral organs in succession. ${ }^{[13]}$ In tubal pregnancy, due to insufficient tissue and blood supply adequate placentation is not possible; most of the pregnancies abort at early stage. Moreover, due to limited distensibility and trophoblastic invasion in the wall of the tube, rupture is very common $(>50 \%)$, resulting in unsuccessful pregnancies. The most common granulomatous infection of the ovary is tuberculosis and usually secondary to tubal disease. ${ }^{14}$ Spread of organisms from the tube might have affected the surface only and produce either an acute or a chronic peri-oophoritis.Often the ovarian substance was involved subsequently. The ovaries are typically involved as tubo-ovarian mass, which is frequently adherent to the omentum and intestine..$^{15}$

Extensive ulceration occurs in severe cases of cervical tuberculosis and reactive atypical hyperplasia of the cryptal epithelium with florid papilla formation develops; the features can resemble carcinoma macroscopically and microscopically. ${ }^{16}$

Li Z et al used three pairs of oligonucleotide primers to differentiate between mycobacterium tuberculosis and nontuberculous mycobacteria by triplex-PCR from formalin fixed, paraffin embedded tissue. ${ }^{17}$ PCR was

Table 1. Tuberculosis of different anatomical sites and percentage $(n=68)$

\begin{tabular}{lcccc}
\hline Site & Number of cases & Percentage (\%) & Caseation present & AFB present in tissue section (ZN stain) \\
\hline a)Cervix & 04 & 05.88 & - & - \\
i) Endocervix & 03 & 04.41 & - & - \\
ii) Ectocervix & 01 & 01.47 & - & 01 \\
b) Endometrium & 38 & 55.88 & - & - \\
i) Proliferative & 31 & 45.58 & 01 & 01 \\
ii) Secretory & 04 & 05.88 & - & - \\
iii) Atrophic & 03 & 04.41 & 05 & 02 \\
c) Fallopian tube & 16 & 23.53 & 01 & - \\
i) Bilateral & 13 & 19.11 & 04 & 02 \\
ii) Unilateral & 03 & 04.41 & 03 & 01 \\
d) Ovaries & 10 & 14.71 & 02 & 01 \\
i) Bilateral & 06 & 08.82 & 01 & - \\
ii) Unilateral & 04 & 05.88 & 09 & 04 \\
Total & 68 & 100 & 01 & - \\
\hline
\end{tabular}




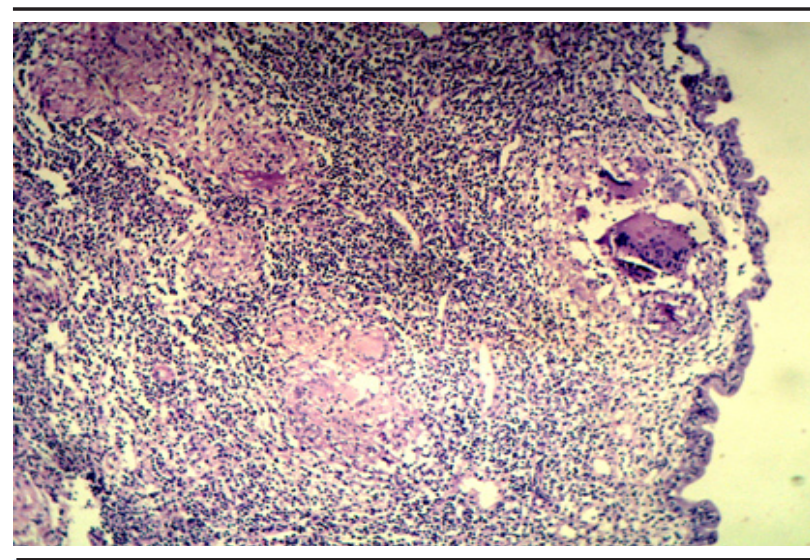

Figure 1. Photomicrograph showing numerous noncaseating epithelioid cell granulomas and Langhans giant cells in functionalis layer of endometrium [HandE $\mathrm{X} 100]$. Inset shows high power view of a typical granuloma in endometrium [HandE $x$ 400].

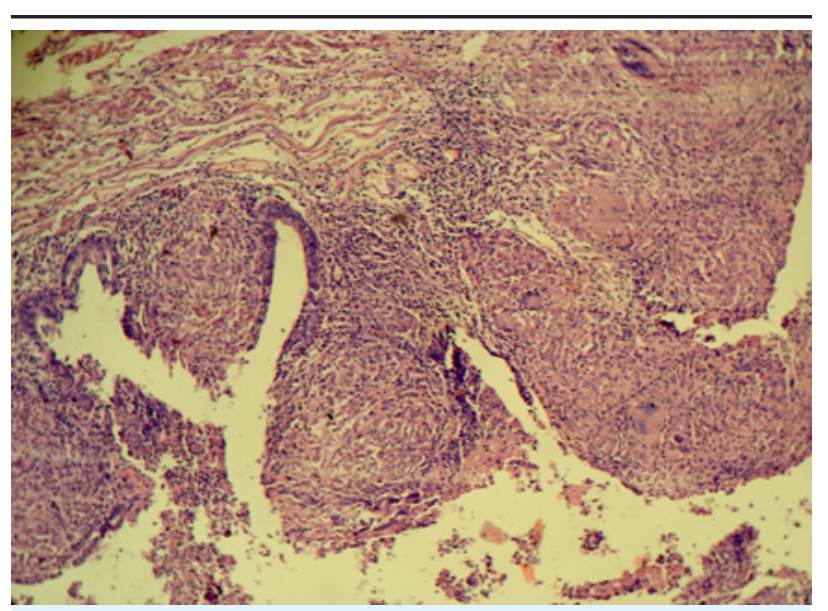

Figure 2. Photomicrograph showing tuberculous salpingitis.Few epithelioid cell granulomas and surrounding dense lymphocytic infiltrate are present in the lamina propria of the plica [HandE $x$ 100].

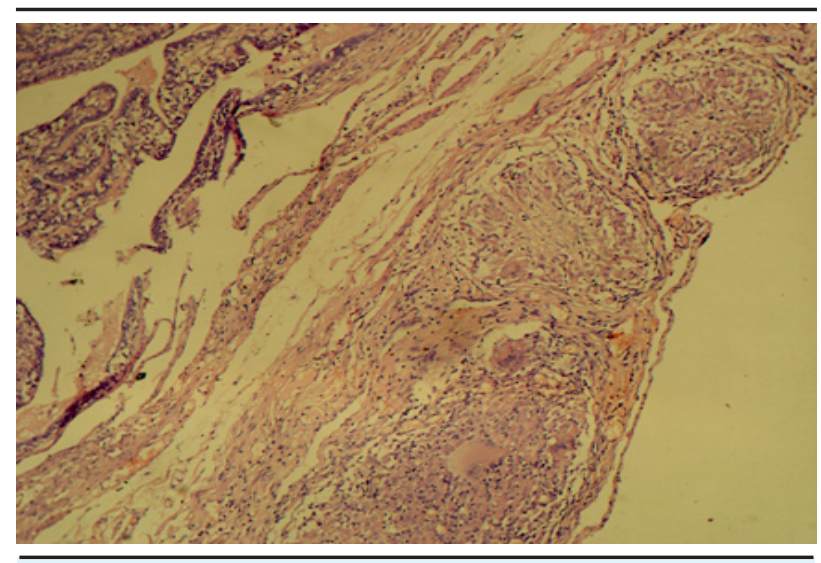

Figure 3. Photomicrograph showing multiple epithelioid cell granulomas in subserosal layer of fallopian tube; an uncommon site for tuberculous involvement [HandE $\times 100]$.

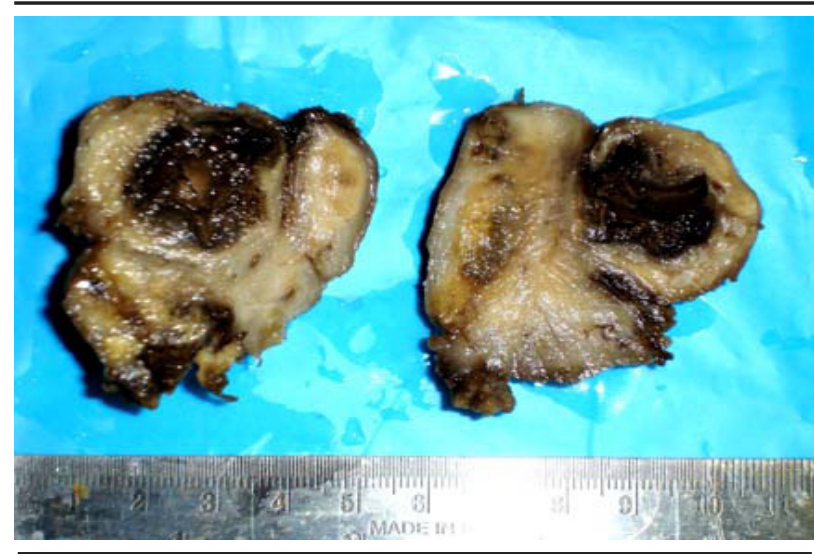

Figure 4. Gross photograph showing tuberculous inflammation which presented as Tubo- Ovarian mass. Multiple small nodular lesions were found in fallopian tube and ovary on cut section.

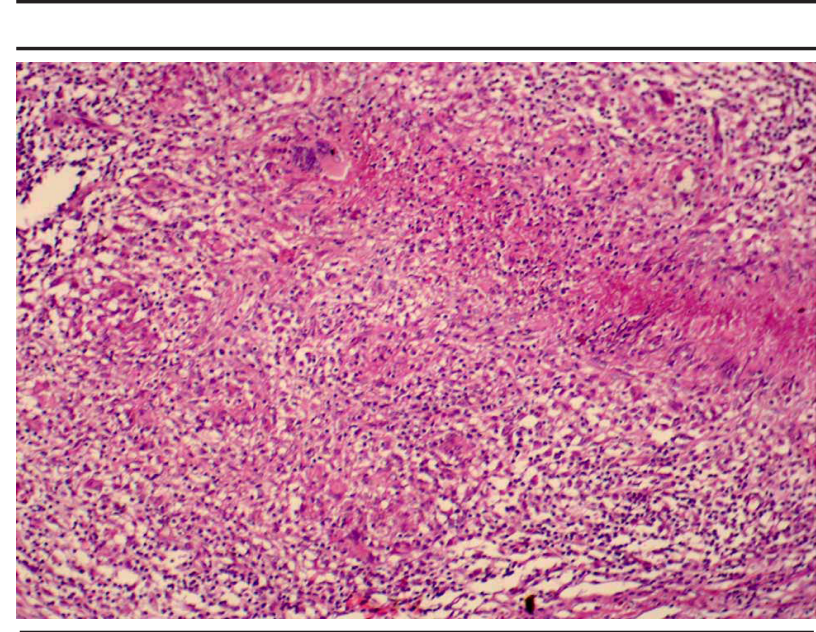

Figure 5. Photomicrograph showing a caseating epithelioid cell granuloma in the ovary [HandE x400].

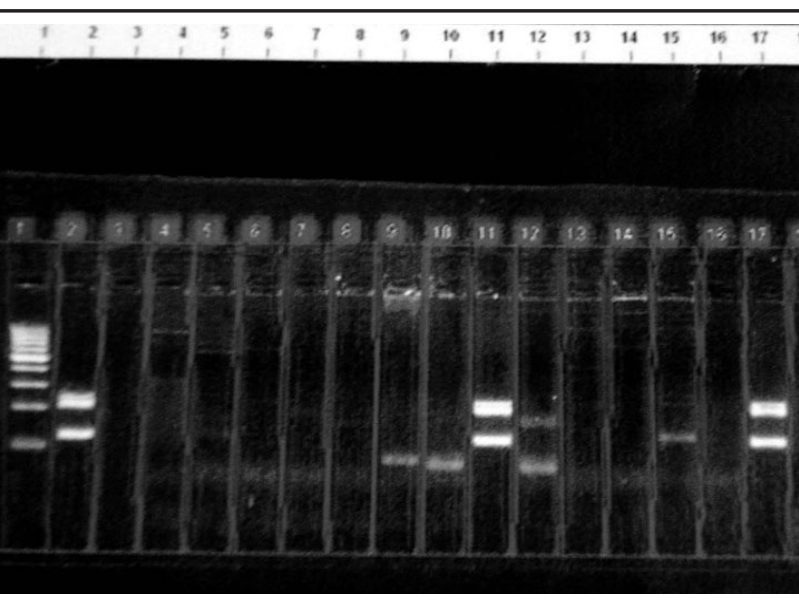

Figure 6. Agarose gel electrophoresis showing PCR-based detection of DNA of Mycobacterium tuberculosis. Lane No.1: marker panel, Lane No. 2: Positive control (123 and 383 bp band), Lane No.3: Negative control, Lane No.11and17: positive test results. 
Mondal et al. A Ten year Clinicopathological Study of Female Genital Tuberculosis and Impact on Fertility

found to have a much higher positivity than conventional test and BACTEC culture ( $P<0.05)$. The primer specific for amplifying the $123 \mathrm{bp}$ IS6110 fragment gave the highest positivity (83\%), followed by $65 \mathrm{k} \mathrm{Da}, 38 \mathrm{k} \mathrm{Da}$ and 85 B RT-PCR in descending order. ${ }^{18}$

Enough prospective data is not available on optimal management of female genital TB. In our patients, six months of anti TB treatment was given in which pyrazinamide was included for the first 2 months. ${ }^{10}$ Sutherland AM advocated for 8 drug programmes and concluded that best results being obtained with streptomycin, para-aminosalicyclic (PAS) acid and isoniazid or with rifampicin, ethambutol and isoniazid. ${ }^{5}$ Indications for surgery in this study included recurrence of bleeding or pain and persistence of pelvic mass which indicated medical treatment failure. Surgery should be done at least 6 weeks after medical treatment, because antimicrobial treatment facilitates the surgical procedure and reduces the risk of perioperative complications. Pregnancy occurred when treatment employed immediately after detection of female genital TB and when no irreversible anatomical pathology was evident.
Chances of having a normal pregnancy were unlikely and the risk of having an ectopic pregnancy was high.

\section{CONCLUSIONS}

Genital tuberculosis is an important cause of female infertility, especially in developing countries like India, Nepal, Bangladesh, and Pakistan. Any woman suspected of genital tuberculosis clinicoradiologically or by other methods should undergo diagnostic workup. If epithelioid granulomas, suspicious of tuberculosis are found on histologic examination, presence of Mycobacterium tuberculosis should be confirmed by culture of tissue and/or fluid (menstrual/peritoneal). Molecular diagnosis by PCR is very useful in culture negative cases. In case of suspected endometrial TB, biopsy for histologic examination should be taken just before the menstrual phase to get highest possibility of detecting tuberculoid granulomas in endometrium. Caseation is uncommon in endometrial TB, and absent in cervical TB. Successful uterine pregnancy is rare after treatment and chance of ectopic pregnancy is high.

\section{REFERENCES}

1. Nwachokor FN, Thomas JO. Tuberculosis in Ibadan, Nigeria - a 30 year review. Cent Afr J Med 2000; 46:287-92.

2. Gupta N, Sharma JB, Mittal S, Singh N, Misra R, Kukreja M. Genital tuberculosis in Indian infertility patients. Int J Gynaecol Obstet 2007; 97:135-8.

3. Qureshi RN, Samad S, Hamid R,Lakha SF. Female genital tuberculosis revisited. J Pak Med Assoc 2001;51:16-8.

4. Namavar Jahromi B, Parsanezhad ME, Ghane-Shirazi R. Female genital tuberculosis and infertility. Int J Gynaecol Obstet 2001;75:269-72.

5. Sutherland AM. Gynaecological tuberculosis since 1951. J Obstet Gynaecol 1997; 17:119-22.

6. Schaefer G. Female genital tuberculosis. Clin Obstet Gynecol 1976; 19:223-239.

7. Chowdhury NN. Overview of tuberculosis of the female genital tract. J Ind Med Assoc 1996; 94:345-6,361.

8. 8. Jedberg H. Study on genital tuberculosis in women. Acta Obstet Gynecol Scand 1950; 1:1-176.

9. Falk V, Ludviksson K, Agren G. Genital tuberculosis in women. Analysis of 187 newly diagnosed cases from 47 Swedish hospitals during the ten-year period 1968 to 1977. Am J Obstet Gynecol 1980; 138:947-7.

10. Gungorduk K, Ulker V, Sahbaz A, Ark C, Tekirdag AI. Postmenopausal Tuberculosis Endometritis. Infect Dis Obstet Gynecol 2007; 2007:27028.
11. Sutherland AM. Postmenopausal tuberculosis of the female genital tract. Obstetrics and Gynecology 1982; 59:553-556.

12. Roy A, Mukherjee S, Bhattacharya S, Adhya S, Chakraborty P. Tuberculous endometritis in hills of Darjeeling: a clinicopathological and bacteriological study. Indian J Pathol Microbiol 1993; 36:361-369.

13. Nogales-Ortiz F, Taracon I, Nogales FF Jr. The pathology of female genital tuberculosis: a 31-year study of 1436 cases. Obstetrics and Gynecology 1979; 53:422-428.

14. Mc Cluggage WG, Allen DC. Ovarian granulomas: a report of 32 cases. Journal of Clinical Pathology 1997; 50:324-327.

15. Yapar EG, Ekici E, Karasahin E, Gokmen O. Sonographic features of tuberculous peritonitis with female genital tract tuberculosis. Ultrasound Obstet Gynecol 1995; 6:121-5.

16. Chakraborty P, Roy A, Bhattacharya S, Adhya S, Mukherjee S. Tuberculous cervicitis: a clinicopathological and bacteriological study. J Ind Med Assoc 1995; 93:167-168.

17. Li Z, Qin W, Yue Q, Meng K, Lin Q. Study on detection of the Mycobacteria DNA in formalin-fixed, paraffin-embedded tissue samples by triplex polymerase chain reaction. Wei Sheng Wu Xue Bao 2002; 42:69-75.

18. Negi SS, Anand R, Pasha ST, Gupta S, Basir SF, Khare S, Lal S. Dignostic potential of IS6110, $38 \mathrm{kDa}$, and 85B sequence-based polymerase chain reaction in the diagnosis of Mycobacterium tuberculosis in clinical samples. Indian Journal of Medical Microbiology 2007; 25: 43-49. 